\title{
Manifold reconnection in chaotic regimes
}

\author{
G. Corso \\ Department of Electrical Engineering and Computer Science and the Electronics Research Laboratory, \\ University of California at Berkeley, Berkeley, California 94720-1770

\section{F. B. Rizzato} \\ Instituto de Física Universidade Federal do Rio Grande do Sul, P.O. Box 15051, 91501-970 Porto Alegre, RS, Brazil
}

(Received 26 November 1997; revised manuscript received 11 August 1998)

\begin{abstract}
In this paper we extend the concept of separatrix reconnection into chaotic regimes. We show that even under chaotic conditions one can still understand enhanced diffusion in phase space in terms of relatively smooth rearrangements of stable and unstable manifolds of unstable fixed points. [S1063-651X(98)04112-9]

PACS number(s): $05.45 .+\mathrm{b}$
\end{abstract}

Two-dimensional nonmonotonic conservative maps are recognized to be of relevance in modeling a number of nonlinear systems, for instance, laser acceleration of charged particles [1-6], and the nonlinear flow of magnetic field lines in fusion machines such as tokamaks and others [7,8]. As opposed to more traditional monotonic versions, nonmonotonic maps are characterized by frequency curves that are not monotonic functions of the action variable [9]. In laser accelerators, nonmonotonicity arises as a result of the relativistic mass variation of the accelerating particles [5]; in tokamaks, it arises as a result of the geometrical peculiarities of the relevant background magnetic fields. In any case, nonmonotonicity has a strong influence on the types of bifurcations that can occur in the associated nonlinear dynamics.

Period doubling cascades of periodic orbits generally precede a transition to chaotic regimes of these orbits, but tangent bifurcations bear no direct relationship to nonintegrability. Indeed, it has been argued that some of the effects preceding a tangent bifurcation even become meaningless in nonintegrable regimes [1]. To analyze the subject, consider the process depicted in the integrable case of Fig. (1) [2]. Two chains of fixed points undergo a reconnection, starting from the leftmost panel. Before a tangent bifurcation where elliptic fixed points collapse against hyperbolic points, the separatrices defining the upper chain undergo a reconnecting process with those defining the lower chain - this is seen in Fig. 1(b). It is precisely due to the smoothness caused by integrability that the reconnection can be seen so clearly. This is why reconnection is thought to be of more relevance in integrable cases. In contrast, the process is generally regarded as of little significance in chaotic regimes because in those situations all separatrices-which shall be correctly called stable and unstable manifolds-would be already interlaced with little global response as relevant control parameters are varied. Speaking in more precise terms, effects associated with reconnections are thought to be unobservable when the elliptic fixed points of the reconnecting chains undergo full cascades of period doublings, before any sort of mutual contact of the relevant manifolds takes place.

While reconnections, as defined in Ref. [1], do not occur in fully chaotic regimes, topological rearrangements of stable and unstable manifolds are possible. We shall use the term "manifold reconnection" to describe such processes. What happens is that even in chaotic regimes the unstable or stable manifolds of originally hyperbolic points may still make a transition from a situation where their mutual crossings are absent-or, actually, relatively infrequent-to a situation where the mutual crossings become very frequent. We shall illustrate the process with a set of figures from which one will be able to see that this change in topology is reminiscent of the corresponding behavior of regular regimes. The macroscopic result of this type of transition is that as soon as the crossings become frequent, stochastic diffusion undergoes an enhancement from slower to faster rates.

From this point on, the discussion relies on more technical grounds. Therefore here we introduce the model map we shall be working with. The map is called the nonmonotonic twist map, and reads

$$
p_{n+1}=p_{n}-k \sin \phi_{n}, \quad \phi_{n+1}=\phi_{n}+f\left(p_{n+1}\right),
$$

where $(p, \phi)$ is a pair of discrete canonical variables- $p$ representing an action and $\phi$ a $2 \pi$-periodic angular coordinate-and where the map is sympletic. Function $f(p)$ is of foremost importance here. It is in fact a measure of the frequency with which the discrete orbits move on the $(p, \phi)$ phase space. In standard monotonic maps it reads $f(p)=p$, but, as we wish to incorporate nonmonotonic features, we add a quadratic term such that $f$ becomes $f(p)=p-\alpha p^{2}$ as in Ref. [1]. In this case one has effectively a nonmonotonic frequency curve with maximum located at $p=1 /(2 \alpha)$, where $d f(p) / d p=0$. Map (1) has several families of fixed points. Let us focus here on the first order family (period 1 orbits) which is characterized by $p_{n+1}=p_{n}$ and $\phi_{n+1}=\phi_{n}+2 m \pi$, with $m$ an arbitrary positive or negative integer; we shall refer to the fixed points as $\left(p_{m}^{*}, \phi_{m}^{*}\right)$. Equation (1) informs us that the fixed points are located at $\phi_{m}^{*}=0, \pi$ and $p_{m}^{*}=[1$ $\pm \sqrt{1-8 m \pi \alpha} /(2 \alpha)]$. In this case of period 1 orbits, let us have a brief look at the distribution of the various fixed points over the phase space. For $m=0$ one has four points located at $p_{o}^{*}=0,1 / \alpha$. If $m>0$ the fixed points lie in the finite interval $0<p<1 / \alpha$, and if $m<0$ the points lie in any of the intervals $-\infty<p<0$ or $1 / \alpha<p<+\infty$. The existence of points located in the finite interval must satisfy the condition $m<1 /(8 \pi \alpha)$, so it may well happen that no fixed point can actually be found there if $\alpha$ is large enough. What hap- 
(a)

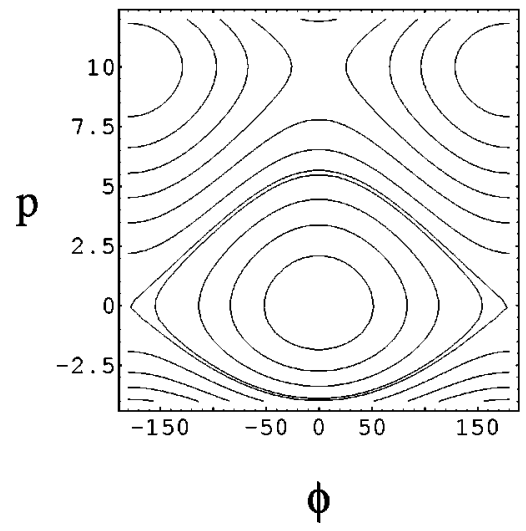

(b)

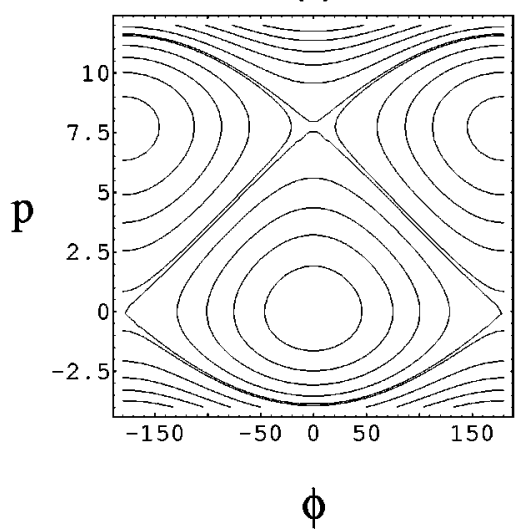

(c)

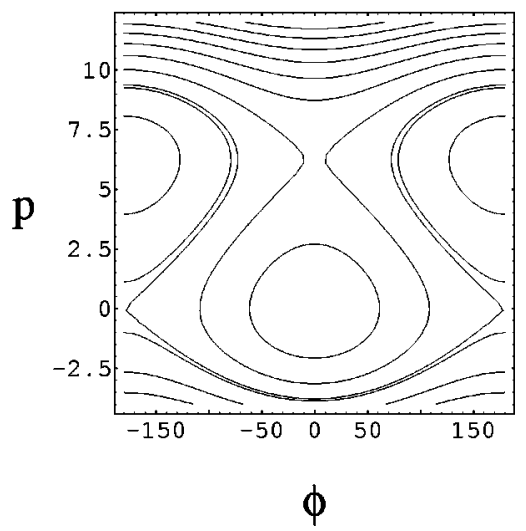

FIG. 1. Reconnection in a purely integrable case. The figure is constructed with an integrable one-degree-of-freedom Hamiltonian of the type $H=p^{2} / 2-\alpha p^{3} / 3-k \cos \phi$ with $(p, \phi)$ as continuous canonical variables; $k=5$ and $\alpha=0.10$ in (a), 0.129 in (b), and 0.16 in (c). $p$ is nondimensional, and $\phi$ is given in degrees.

pens is that as $\alpha$ grows from some small value, all the fixed points originally located in the subinterval $0<p<1 /(2 \alpha)$, collapse, at $p=1 /(2 \alpha)$, against the corresponding points originally located in the subinterval $1 /(2 \alpha)<p<1 / \alpha$, via a sequence of inverse tangent bifurcations. Meanwhile, the points placed externally to the interval $(0,1 / \alpha)$, as well as the points of the pair $m=0$, simply approach each other but never touch. We point out that although the latter two types of fixed points never undergo an inverse tangent bifurcation, their manifolds can naturally undergo reconnection processes. In previous works [1] the $m \geqslant 1$ case has been investigated. It has been shown that when chaos is absent, in the sense that elliptic points of the various chains have not yet period doubled to chaos, initial conditions at negative values of $p$ do not move up to positive values unless the separatrix layers of the two chains corresponding to the $m=1$ resonances touch each other. In addition, it has been argued that for those situations where the elliptic fixed points have already undergone full cascades of period doublings, reconnection becomes a meaningless concept [1]. We now proceed to show that the concept can still be useful in those cases; even under chaotic conditions, some noticeable effects resulting from manifold rearrangements can be in fact observed. Specifically, we shall show that after what could be best called a reconnectionlike process, diffusion is enhanced from low to higher values.

Let us focus the discussion on the $m=0$ case, because this resonance is the largest one in the system. The linear stability of the fixed points can be examined from the eigenvalue of the linearized map around the fixed points, $\lambda=[(2-\kappa)$ $\left.\pm \sqrt{(\kappa-2)^{2}-4}\right] / 2$, with $\kappa \equiv \cos \left(\phi_{o}^{*}\right)\left(1-2 \alpha p_{o}^{*}\right) k$. For a real $k$ (complex), the corresponding fixed point is unstable (stable). One then sees that for the chain located at $p_{o}^{*}=0$, the fixed point at $\phi_{o}^{*}=\pi$ is always unstable, while that at $\phi_{o}^{*}=0$ is unstable only when $k>4$, being stable for $0<k<4$. As for the chain at $p_{o}^{*}=1 / \alpha$, the point at $\phi_{o}^{*}=0$ is always unstable, while that at $\phi_{o}^{*}=\pi$ is unstable when $k>4$; in both cases, destabilization occurs via period doubling of the elliptic points. One can also make an estimate of the condition to be observed for separatrix touching. To do so, let us first imagine that we are working in a situation where $k$ is small and the dynamics is therefore mostly regular. What happens then is that the advance within any particular resonant island tends to be slow. One can thus approximate $p_{n+1}-p_{n}$ and $\phi_{n+1}-\phi_{n}$ by their respective infinitesimal increments $d p$ and $d \phi$, and finally write an expression valid in the vicinity of a $m=0$ chain: $d p / d \phi=-k \sin (\phi) / f(p)$. Then, with obvious notation, one obtains an expression for the separatrix of the lower chain:

$$
\int^{p_{\mathrm{sep}}} f(p) d p=k\left(1+\cos \left(\phi_{\mathrm{sep}}\right)\right)
$$

The upper separatrix of the $\left(p_{o}=0, \phi_{o}=\pi\right)$ fixed point touches the $\left(p_{o}=1 / \alpha, \phi_{o}=0\right)$ fixed point of the corresponding upper chain (see Fig. 1) when

$$
k=k_{t} \equiv 1 /\left(12 \alpha^{2}\right)
$$

such a value for $k$ is also known as the reconnection threshold in regular regimes. We can now draw Fig. 2. The figure is similar to the corresponding figures shown in Refs. [1,4]. It simultaneously displays the threshold and period doubling curves in the parameter space, with the threshold curve extended into the chaotic regime. The period doubling curve, in the $m=0$ case analyzed here, is simply a horizontal line at $k=4$. It is thus seen that, for values of $\alpha$ below $\alpha \sim 0.144$,

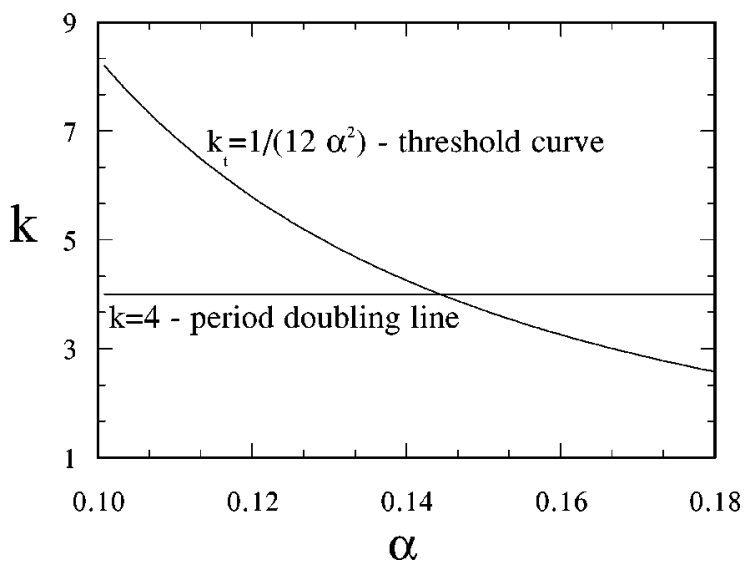

FIG. 2. Parameter space $(\alpha, k)$ for the period $1 m=0$ resonance, and the relevant threshold curves. 
(a)

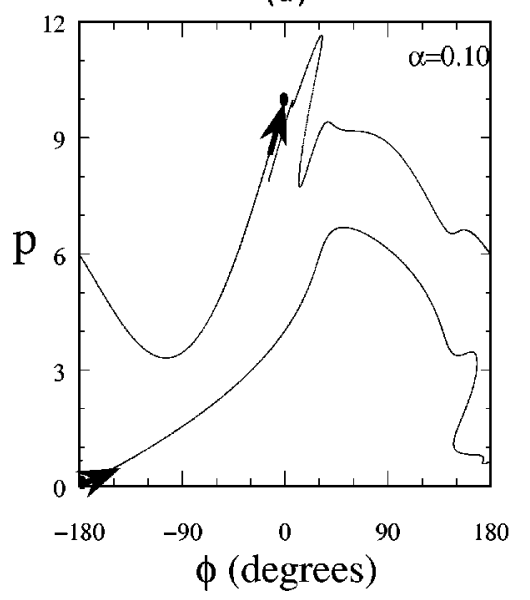

(b)

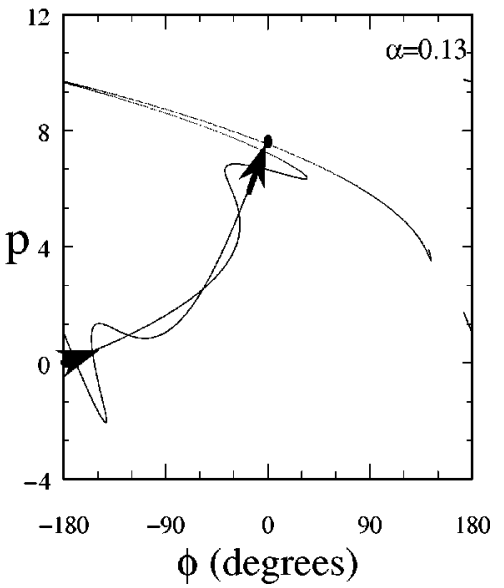

FIG. 3. The rearrangements of stable and unstable manifolds in a deeply chaotic regime for which $k=5$. period doublings occur before reconnection. Previous works have focused interest on the region $\alpha>0.144$, because in that region one could clearly speak in terms of reconnectionrecall that in this region reconnection takes place before period doublings. In the present paper we shall concentrate efforts to see what happens deep into chaotic regimes when $\alpha<0.144$.

To start the investigation, let us consider the vicinity of the threshold curve at $k=5$. For this value of $k$, elliptic points have been totally destroyed by full cascades of period doublings. Therefore, as we increase $\alpha$, the theoretical reconnection threshold can be attained while the system remains in a deep chaotic regime. Let us try to examine how the relevant manifolds of the $m=0$ resonances behave in phase space. The analysis is made with the help of the panels of Fig. 3, where we focus attention on the upper unstable and lower stable manifolds (respective orientations indicated by arrows) of the originally hyperbolic points (points indicated by black dots) of the lower and upper chains, respectively; in order to draw the manifolds, we launch 1000 initial conditions along the linearized manifolds, iterating the dynamics forward or backward according to the case.

First of all we note that in integrable cases, separatrices describe homoclinic loops. Now, even in our nonintegrable case, when $\alpha$ is small it is seen that the tendency of the unstable manifold of the lower chain is to follow the homoclinic loop of the integrable approximation. Of course, due to the nonintegrable features, the unstable manifold eventually starts to execute increasingly large oscillations after it first intersects the stable manifold of the same point. As the orbit is about to complete the homoclinic loop the oscillations grow, and as the oscillations grow it may happen that the unstable manifold of the lower chain crosses the stable manifold of the upper chain as well. But what must be observed here is that this latter intersection occurs only after the stable manifold of the lower chain crosses the unstable manifold of the same lower chain many times. In this situation one can look at the process as resembling the integrable case, although, as mentioned before, one is in a deeply chaotic regime.

Then, when one increases the value of $\alpha$, the overall topology of manifold crossings appears to undergo a substantial change. This can be observed in Fig. 3(b), where it is seen that this change in topology is in fact very similar to what happens in the purely integrable model. Here the un- stable manifold of the hyperbolic point of the lower chain makes a direct connection with the stable manifold of the upper chain. This leads to the opening of a new diffusive channel connecting the regions located below the lower chain and above the upper chain. In other words, although one is in a deep chaotic regime since the original elliptic points have fully period doubled to chaos, noticeable changes can be expected as a result of clear alterations on the topology of the manifolds. This behavior of the manifolds is rather conservative in the sense that while the elliptic points have bifurcated, the manifolds still try to preserve some aspects of integrability. It appears that this feature takes place because, near the midpoint between the two chains, the orbital frequency attains an extremum $d f(p) / d p=0$ in view of nonmonotonicity. As a result, the local dynamics is relatively linear, and nonintegrable effects are relatively smaller than in other regions of the phase-space.

We now proceed to show that reconnections in chaotic regimes may have a direct influence on macroscopic processes such as diffusion. We actually measure a fraction of particles that are transmitted across the region where $d f(p) / d p \sim 0$ in a numerical experiment that goes as follows.

A set of 1000 initial conditions is launched in the region below the lower reconnecting chain, and iterated many times. All particles arriving at the region above the upper

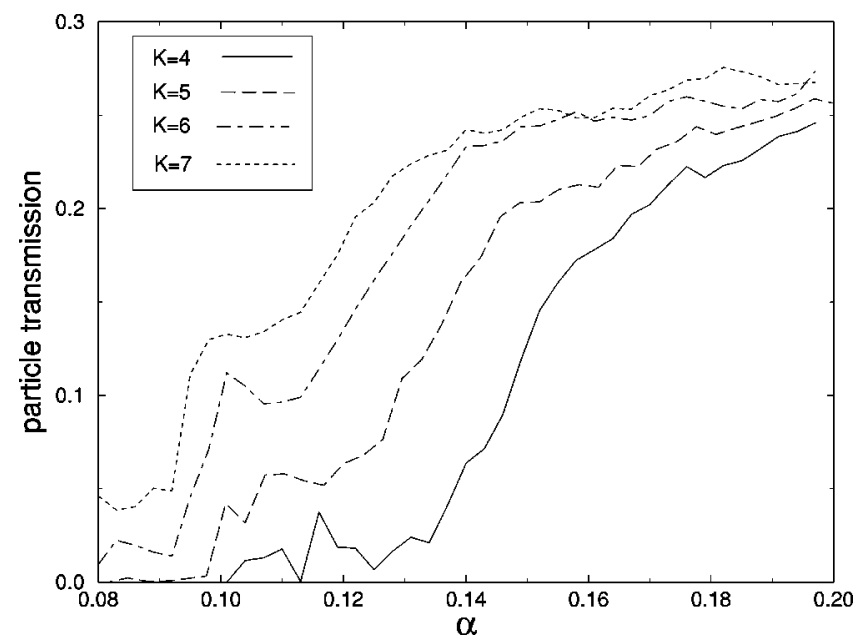

FIG. 4. Transmission fraction as a function of $\alpha$ in chaotic regimes. 
(a)

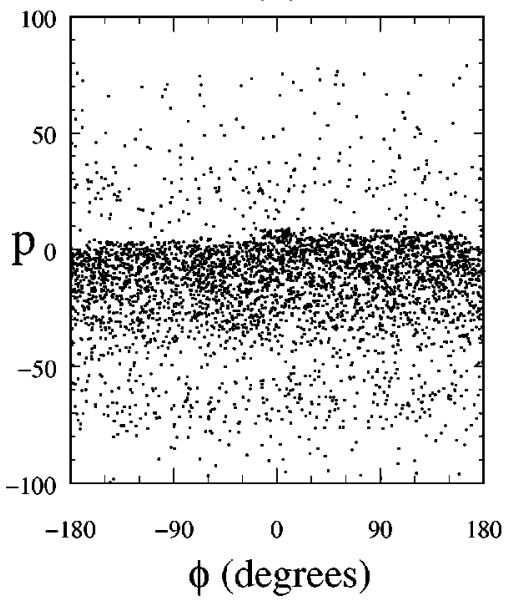

(b)

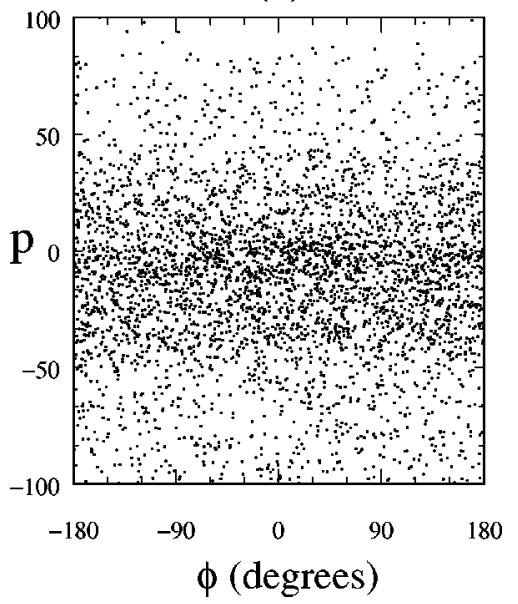

FIG. 5. Poincaré plot for $k$ =5. $\alpha=0.10$ in (a) and 0.16 in (b). reconnecting chain are reinjected into the initial lower region, to create a steady state in the long run. The simulation is optimized by reflecting particles that move into the region below the injection momentum $p=-2.0$. The mental picture one can form of the system is that of a multicomponent gas placed in a vessel divided by a semipermeable membrane whose role is played by the reconnecting chains. The gas is placed below the lower chain, and some of the particles are able to cross over up to the region above the upper chain. As time evolves, one reaches a steady state, and we measure transmission by computing the number of particles in the upper region divided by the total number of particles.

Figure 4 shows the fraction of particles transmitted after many iterations ( $\sim 1300$ iterates), versus $\alpha$, for $k=4,5,6$, and 7 . In this range of $k$, as suggested by Fig. 2, there is no $K A M$ curve in the phase space, except in the case $k=4$; even in this case, however, all the separatrix layers are already chaotic and our discussion applies. In Fig. 4, we see an increase in particle transmission starting at a value of $\alpha$ near the one corresponding to the integrable reconnection. The reconnecting value of $\alpha$ can be evaluated analytically using Eq. (2). For $k=4,5,6$, and 7, one obtains $\alpha=0.144,0.129$, 0.118 , and 0.109 , respectively. It is also observed that the transmission reaches a plateau after an ascending stage; presumably this happens when the reconnection is fully completed. Note that the transmission curves gradually displace leftward as one increases the value of $k$. This kind of behavior is embodied in relation (3), from which is seen that larger $k$ 's imply smaller $\alpha$ 's. We note that the ascending range of both curves are relatively smooth and are not sharply located at the reconnecting value of $\alpha$, since this critical number is calculated within an integrable approximation.
In Fig. 5, we finally display the surface of sections of 20 trajectories initially placed at $p=-2.0$ with a uniform distribution along $\phi$. Figure 5(a) shows a value for $\alpha$ prior to the reconnection, $\alpha=0.10$, and Fig. 5(b) a value for $\alpha$ past the reconnection threshold, $\alpha=0.16$. The alterations in the diffusive pattern suggested by all the previous analysis can be seen in those figures as well, while in Fig. 5(a) the particles remain mostly in the lower region. In Fig. 5(b) particles can be easily transmitted across the barrier at $p \sim 0$.

To summarize, in this paper we have investigated the effect that reconnections involving unstable manifolds of deeply chaotic regimes can have on some macroscopically observable features like particle diffusion. We have used a nonmonotonic map to create reconnecting chains. Examining a particular family of fixed points for which $m=0$ in the notation of the text, we have seen that the topology of manifolds of unstable fixed points may have a similar behavior to that in an integrable approximation. As we vary convenient parameters, manifolds of different chains of islands can be clearly seen to undergo a rearrangement process even in deep chaotic regimes.

F.B.R. would like to acknowledge the hospitality at the Department of Electrical Engineering and Computer Science, U.C. at Berkeley, where part of this work was developed. We deeply thank Professor Allan Lichtenberg for his enlightening suggestions on the paper. G.C. was supported by the Coordenadoria de Aperfeiçoamento de Pessoal de Nível Superior (CAPES), Brazil, and we received additional support from Financiadora de Estudos e Projetos (FINEP) and Conselho Nacional de Desenvolvimento Científico e Tecnológico (CNPq), Brazil.
[1] J. E. Howard and S. M. Hohns, Phys. Rev. A 29, 418 (1984).

[2] R. E. de Carvalho and A. M. O. de Almeida, Phys. Lett. A 162, 457 (1992).

[3] D. Farina and R. Pozzoli, Phys. Rev. A 45, 575 (1992).

[4] J. E. Howard and J. Humphreys, Physica D 80, 256 (1995).

[5] G. Corso and F. B. Rizzato, Phys. Rev. E 52, 3591 (1995); Physica D 80, 296 (1995).

[6] A. J. Lichtenberg and M. A. Lieberman, Regular and Chaotic
Dynamics (Springer, Berlin, 1991).

[7] G. A. Oda and I. L. Caldas, Chaos Solitons Fractals 5, 15 (1995).

[8] G. Corso, G. A. Oda, and I. L. Caldas, Chaos Solitons Fractals 8, 1891 (1997).

[9] D. del-Castillo-Negrete, J. M. Greene, and P. J. Morrison, Physica D 91, 1 (1996); 100, 311 (1997). 